\title{
BMR in children with chronic kidney disease
}

\author{
C. E. Anderson ${ }^{1}$, R. Gilbert ${ }^{1}$ and M. Elia ${ }^{3}$ \\ ${ }^{1}$ Department of Nutrition and Dietetics, ${ }^{2}$ Child Health and ${ }^{3}$ Institute of Human Nutrition, Southampton General Hospital, \\ Southampton SO16 6YD, UK
}

The data on the effect of chronic kidney disease in children on BMR are conflicting, confusing and limited. This causes difficulty in calculating energy requirements. The aim of the present study was to 1) examine the extent to which BMR in children with CKD differs from that of healthy children and 2) to determine whether resting energy expenditure in a group of children with CKD differs from that of healthy children.

The present study involved twenty children with chronic renal failure and twenty control children who did not differ in age (years; 12.1 (SD 3.5) v. 11.8 (SD 3.3)), (weight (kg); 40.1 (SD 14.5) v. 46.3 (SD 16.8)) or (height (m); 1.46 (SD 0.2) v. 1.50 (SD 0.18)). Glomerular filtration rate (GFR) in the renal group was 34 (SD 19.9) $\mathrm{ml} / \mathrm{min}$ per $1.73^{2}$. Z scores were significantly different for weight ( -0.29 (SD 1.15 ) v. 0.66 (SD 0.99); $P=0.007$ ), and BMI ( -0.99 (SD 1.1) v. 0.65 (SD 1.0) $P=0.031$ ) but not height $(-0.32$ (SD 1.22) v. (0.35 (SD 1.35)).

BMR was measured by indirect open circuit calorimetry using Deltatrack II (Datex-Ohmeda, Helsinki, Finland). Children were asked to fast overnight for $12 \mathrm{~h}$ and then rest for $30 \mathrm{~min}$ in a recumbent position prior to measurements of gaseous exchange. Readings were taken for $30 \mathrm{~min}$ in a quiet room with an ambient temperature of approximately $23^{\circ} \mathrm{C}$. Energy expenditure was calculated using the equation of Elia and Livesey ${ }^{(1)}$. Predicted BMR was calculated using the Schofield equations for age, gender, weight and height ${ }^{(2)}$. The dry weight of children with chronic kidney disease was estimated by clinical examination by a consultant paediatric nephrologist. GFR was calculated using the Schwartz formula ${ }^{(3,4)}$.

The results show (1) GFR was significantly related to BMR $\left(R^{2}=0.261, R=0.511, P=0.021\right)$ so that at a GFR of 0 BMR was $91 \%$ of predicted BMR and at a GFR of 70 BMR was $112 \%$ of predicted BMR. This could not be explained by nutritional status. Thus the significance of this relationship was 0.021 before adjustment for $\mathrm{Z}$ scores weight and height and 0.024 after adjustment. (2) There was no significant difference in measured BMR (kJ/d) between the renal and healthy groups either before (5431 (SD 1297) v. 5544 (SD 745 ); $P=0.736$ ) or after adjustment for age and gender (5435 (SE 192) v. 5586 (SE 197); $P=0.588$ ). BMR, as a percentage of Schofield predicted values, also did not differ between groups (100 (SD 13) v. 99 (SD 13); $P=0.768$ ).

In conclusion, although the BMR of this group of children with chronic renal failure did not differ from that of normal children, this was lower in more severe renal disease (lower GFR), and needs consideration when estimating the energy requirements of children with severe chronic renal failure whose growth is of concern. Consideration also needs to be given to the effects of CKD on total energy expenditure, which includes physical activity.

The Health Foundation funded this project.

1. Elia M \& Livesey G (1992) World Rev Nutr Diet 70, 68-131.

2. Schofield WN (1985) Clin Nutr 39C, Suppl. 1, 5-41.

3. Schwartz GJ, Haycock GB, Edelmann CM Jr et al. (1976) Pediatrics 58, 259-263.

4. K/DOQI (2000) Clinical Guidelines for Chronic Kidney Disease: Evaluation, Classification, and Stratification. www.kidney.org/Professionals/Kdoqi/ guidelines (accessed June 2005). 